\title{
Geometry of Lax pairs: Particle motion and Killing-Yano tensors
}

\author{
Marco Cariglia* \\ Departamento de Física, Universidade Federal de Ouro Preto, \\ ICEB, Campus Morro do Cruzeiro, Ouro Preto 35400-000, Minas Gerais, Brasil \\ Valeri P. Frolov ${ }^{\dagger}$ \\ Theoretical Physics Institute, University of Alberta, Edmonton, Alberta, Canada T6G 2G7 \\ Pavel Krtouš \\ Faculty of Mathematics and Physics, Institute of Theoretical Physics, Charles University in Prague, \\ V Holešovičkách 2, 18000 Prague, Czech Republic \\ David Kubižnák ${ }^{\S}$ \\ Perimeter Institute, 31 Caroline Street North, Waterloo, Ontario, Canada N2L 2 Y5
}

(Received 22 October 2012; published 2 January 2013)

\begin{abstract}
A geometric formulation of the Lax pair equation on a curved manifold is studied using the phase-space formalism. The corresponding (covariantly conserved) Lax tensor is defined and the method of generation of constants of motion from it is discussed. It is shown that when the Hamilton equations of motion are used, the conservation of the Lax tensor translates directly to the well-known Lax pair equation, with one matrix identified with components of the Lax tensor and the other matrix constructed from the (metric) connection. A generalization to Clifford objects is also discussed. Nontrivial examples of Lax tensors for geodesic and charged particle motion are found in spacetimes admitting a hidden symmetry of KillingYano tensors.
\end{abstract}

DOI: 10.1103/PhysRevD.87.024002

\section{INTRODUCTION}

Since its discovery in 1968 [1], the Lax pair formulation has played an invaluable role in studying the integrability of various systems. Although first formulated for systems with infinite degrees of freedom, the formalism can also be used for, and provides an elegant description of, special finite-dimensional systems with symmetries. Examples of such (completely integrable) systems admitting a Lax pair formulation include the Kepler problem, the Euler, Lagrange, and Kowalevski tops, the Neumann model, and the Toda lattice; we refer the reader to the monograph [2] and to references therein. In what follows we concentrate on finite-dimensional systems. Namely, we shall discuss a geometrization of the Lax pair matrices for motion in curved spacetime.

A standard dynamical system is described on a phase space $P$ equipped with the symplectic two-form $\Omega$ and with the corresponding Poisson brackets $\{$,$\} . The dynamics$ is encoded in a Hamiltonian $H$ through the evolution equation for an arbitrary scalar observable $F$,

$$
\dot{F}=\{F, H\}
$$

\footnotetext{
*marco@iceb.ufop.br

†vfrolov@ualberta.ca

‡Pavel.Krtous@utf.mff.cuni.cz

\$dkubiznak@perimeterinstitute.ca
}

PACS numbers: 02.30.Ik, 04.50.Gh, 45.10.Na, 45.20.Jj

The Lax pair method consists of finding two phase-space valued matrices $L$ and $M$, such that the equations of motion imply the Lax pair equation

$$
\dot{\mathrm{L}}=[\mathrm{L}, \mathrm{M}] \text {. }
$$

The stronger formulation requires that Eq. (1.2) implies the equations of motion, in which case the Lax pair formulation can be used as a starting point of the description of the dynamical system.

However, even without this latter stronger condition, the Lax pair matrices satisfying Eq. (1.2) play an important role in the study of integrability since they allow a simple construction of constants of motion. Indeed, the solution of Eq. (1.2) is of the form $\mathrm{L}(t)=\mathrm{G}(t) \mathrm{L}(0) \mathrm{G}^{-1}(t)$, where the evolution matrix $\mathrm{G}(t)$ is determined by the equation $\dot{\mathrm{G}}=-\mathrm{MG}$. Therefore, if $I(\mathrm{~L})$ is a function of $\mathrm{L}$ and is invariant under conjugation $\mathrm{L} \rightarrow \mathrm{GLG}^{-1}$, then $I(\mathrm{~L}(t))$ is a constant of motion. All such invariants can be generated from the traces of various matrix powers of $L$ :

$$
\operatorname{tr}\left(L^{j}\right) .
$$

The particular Lax pair may not yield all the constants of motion. However, in such a case it is often possible to upgrade the initial Lax pair so that the upgraded one already yields all the conserved observables of the dynamical system. Since the dimensionality of the Lax matrices is not fixed and the Lax pair equation is linear, two Lax pairs can be easily combined by their direct sum. Another useful 
method of producing a parametric class of Lax pairs is to introduce so-called spectral parameters; see, e.g., [2].

Unfortunately, in general there is no constructive procedure to find a Lax pair for the given problem or even to determine whether the Lax pair (in its stronger formulation) exists. Moreover, the solution is in no sense unique and even the dimensionality of the matrices may vary. However, when the Lax pair exists, it can be a very powerful tool for dealing with the conserved quantities.

In this paper, we focus on the construction of the Lax pair for motion on a curved manifold. In this case the phase space $P$ is given as a cotangent bundle of a configuration space $M$. There are thus two important features of such a theory which single it out among generic dynamical systems: (i) it has a preferred splitting of $2 \times D$ phase coordinates into two sets $-D$ spacetime coordinates $x^{a}$ and $D$ momenta $p_{a}$; (ii) the configuration space has an additional structure on it-the metric $g_{a b}$.

The prominent example of such dynamical systems is the motion of particles and light in curved spacetime, which plays an important role in general relativity and its generalizations. We will concentrate mainly on this system. However, the dynamical systems with the cotangent bundle structure also include all nonrelativistic systems which start with the Lagrangian description on the configuration space. The metric on the configuration space in such cases emerges from the kinetic part of the Hamiltonian.

Our aim is to show that for these dynamical systems it is possible to formulate a covariant analogue of the Lax equation. This covariant formulation allows us to employ the description of explicit and hidden symmetries of curved manifolds encoded in structures as Killing vectors, Killing tensors, or Killing-Yano tensors. In particular, for the motion of particles in a curved spacetime we establish a relationship between conserved quantities connected with the Lax pair, and integrals of motion connected with hidden symmetries generated by Killing-Yano tensors.

To achieve this program, in Sec. II we analyze geometrical structures on the phase space with a cotangent bundle structure, especially those induced from a configurationspace-covariant derivative. We define a covariant derivative acting on phase-space fields with configuration-space tensor indices.

With this geometrical background, in Sec. III we formulate the covariant Lax equation,

$$
\frac{\nabla}{d t} L_{b}^{a}=0
$$

for a phase-space-dependent tensor field $L^{a}{ }_{b}(x, p)$ and call this object a Lax tensor. Here, $\frac{\nabla}{d t}$ is the time derivative (the derivative along the Hamiltonian flow) defined in terms of the covariant derivative mentioned above.

By construction, any scalar invariant built covariantly from the Lax tensor (or from a set of Lax tensors) is preserved along phase-space trajectories and thus defines a constant of motion. Moreover, all scalar invariants encoded in one Lax tensor $L^{a}{ }_{b}$ can be generated from the traces of powers of $L^{a}{ }_{b}$,

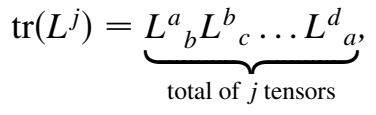

which is an obvious geometric analogue of Eq. (1.3).

There is a direct translation of the covariant Lax equation (1.4) into the standard Lax pair equation (1.2). Namely, the components of the Lax tensor $L^{a}{ }_{b}$ form the Lax matrix $L$, while the other matrix $M$ is given through the (Christoffel) connection symbols as follows:

$$
\mathrm{L}=\left[L_{b}^{a}\right], \quad \mathrm{M}=\left[\frac{\partial H}{\partial p_{n}} \Gamma_{n b}^{a}\right] .
$$

An analogous geometric construction using Clifford matrices will also be introduced. Similar ideas in special cases have already been studied in Refs. [3-7].

The paper is organized as follows. In the next section we review the phase-space formalism for the phase space built as a cotangent bundle of a configuration manifold. In particular, we define the covariant phase-space derivative. This is further elaborated in the Appendix. In Sec. III we recapitulate the Lax pair formalism and derive its geometric covariant counterpart encoded in Eq. (1.4). Section IV illuminates the previous discussion by studying two "trivial" examples of Lax tensors for geodesic motion in generic spacetimes. Section V is devoted to highly nontrivial examples of Lax tensors for geodesic motion in special spacetimes admitting a hidden symmetry of Killing-Yano tensors. The motion of a charged particle in weakly charged KerrNUT spacetimes is discussed in Sec. VI. We conclude in Sec. VII.

\section{COVARIANT DERIVATIVE ALONG A PHASE-SPACE TRAJECTORY}

\section{A. Phase space}

Particle motion in a curved spacetime ${ }^{1} M$ can be described in the language of Hamiltonian mechanics. The phase space $P$ is the cotangent bundle $\mathbf{T}^{*} M$ equipped with the standard symplectic structure $\Omega$ and Poisson brackets. A phase-space point can be written as $[x, p]$ with position $x \in M$ and momentum $p \in \mathbf{T}_{x}^{*} M$. Any spacetime coordinates $x^{a}$ together with the corresponding components $p_{a}$ of the momentum $p$ form the canonical coordinates $x^{a}, p_{b}$ in which the symplectic structure and Poisson brackets read

\footnotetext{
${ }^{1}$ As was mentioned in the Introduction, although we apply our discussion mainly to the relativistic context, the formalism introduced here does not depend on the signature of the metric and it can also be used in the case of standard nonrelativistic mechanics with a curved configuration space. We use small Latin letters for spacetime (configuration space) tensor indices and we drop indices when it does not cause confusion.
} 


$$
\begin{gathered}
\Omega=d x^{a} \wedge d p_{a}, \\
\{F, G\}=\frac{\partial F}{\partial x^{a}} \frac{\partial G}{\partial p_{a}}-\frac{\partial F}{\partial p_{a}} \frac{\partial G}{\partial x^{a}} .
\end{gathered}
$$

Given a Hamiltonian $H$, the time derivative of any observable $F$ is

$$
\dot{F}=\{F, H\},
$$

which can also be interpreted as the derivative along the Hamiltonian flow $X_{H}=\Omega^{-1} \cdot d H$,

$$
\dot{F}=X_{H} \cdot d F \text {. }
$$

More details on the conventions used for the symplectic structure and its inverse can be found in the Appendix.

\section{B. Covariant phase-space derivative}

The derivative (2.4) along $X_{H}$ is defined only for scalar phase-space observables. It is fruitful to generalize it to more general observables, namely to fields on the phase space with spacetime indices. Such fields appear naturally as a combination of spacetime tensors contracted with momenta, e.g.,

$$
A_{b \ldots}^{a \ldots}(x, p)=a_{b \ldots}^{a \ldots}(x)+b_{b \ldots}^{a \ldots k}(x) p_{k}+c_{b \ldots}^{a \ldots k}(x) p_{k} p_{l}+\cdots
$$

One can also consider fields of more complicated analytic form, e.g., $A_{a}(x, p)=\left(g^{k l}(x) p_{k} p_{l}\right)^{-\frac{1}{2}} p_{a}$.

To define a derivative of tensor fields one needs an additional structure. For spacetime fields such a structure is a covariant derivative. Assuming the covariant derivative $^{2} \nabla$ on the spacetime we lift this derivative to act on phase-space fields with spacetime indices. We call the resulting operation a covariant phase-space derivative.

This derivative is defined along a general phase-space direction $X \in \mathbf{T} P$. The direction can be represented using a configuration direction $u \in \mathbf{T} M$ and a momentum direction $f \in \mathbf{T}^{*} M$. The definition of this splitting requires the covariant derivative $\nabla$. Namely, if $X$ is tangent to a phasespace trajectory $[x(t), p(t)]$, its configuration part $u$ is tangent to the configuration trajectory $x(t)$ and the momentum part $f$ is the covariant derivative of $p(t)$ along $x(t)$. That is, we have

$$
f_{a}=\frac{\nabla}{d t} p_{a}=\dot{p}_{a}-u^{n} \Gamma_{n a}^{k} p_{k} .
$$

See Eq. (A22) and Fig. 1 in the Appendix for further discussion.

\footnotetext{
${ }^{2}$ This can be an arbitrary covariant derivative, and is not necessarily the metric one. For simplicity, in the body of the paper we assume vanishing torsion. Expressions with torsion can be found in the Appendix. In the case of geodesic motion we choose the metric covariant derivative.
}

The covariant derivative $\nabla_{X}$ along the direction $X=[u, f]$ acting on phase-space fields with spacetime indices is defined by the following rules:

(i) For a field depending only on the spacetime position, $A_{b \ldots}^{a \ldots}(x, p)=\alpha_{b \ldots}^{a \ldots}(x)$, the derivative reduces to the standard covariant derivative along $u$ :

$$
\nabla_{X} A_{b \ldots}^{a \ldots}=u^{n} \nabla_{n} \alpha_{b \ldots}^{a \ldots} .
$$

(ii) For the momentum field $p_{a}$, the derivative gives the momentum part of $X$ :

$$
\nabla_{X} p_{a}=f_{a}
$$

(iii) The derivative $\nabla_{X}$ satisfies all standard rules for the derivative (i.e., linearity, the Leibniz product rule, and the chain rule).

These rules reflect the splitting of the phase-space direction into configuration and momentum parts. This can also be encoded using the partial derivatives ${ }^{3} \frac{\nabla}{\partial x}$ and $\frac{\partial}{\partial p}$ introduced in the Appendix:

$$
\nabla_{X} A_{b \ldots}^{a \ldots}=u^{n} \frac{\nabla_{n}}{\partial x} A_{b \ldots}^{a \ldots}+f_{n} \frac{\partial}{\partial p_{n}} A_{b \ldots}^{a \ldots .} .
$$

For a phase-space trajectory with tangent field $X$ we naturally write

$$
\frac{\nabla}{d t} A_{b \ldots}^{a \ldots} \equiv \nabla_{X} A_{b \ldots}^{a \ldots}
$$

and call $\frac{\nabla}{d t}$ the covariant derivative along the phase-space trajectory or just the covariant time derivative. The configuration and momentum parts of the Hamiltonian flow $X_{H}$ are

$$
u^{a}=\frac{\partial H}{\partial p_{a}}, \quad f_{a}=-\frac{\nabla_{a} H}{\partial x},
$$

cf. Eq. (A7), which gives

$$
\frac{\nabla}{d t} A_{b \ldots}^{a \ldots}=\frac{\partial H}{\partial p_{n}} \frac{\nabla_{n}}{\partial x} A_{b \ldots}^{a \ldots}-\frac{\nabla_{n} H}{\partial x} \frac{\partial}{\partial p_{n}} A_{b \ldots}^{a \ldots .}
$$

This is a natural generalization of Eq. (2.3) to the case of tensor-valued observables.

The introduced phase-space covariant derivative can be expressed in coordinates,

$$
\nabla_{X} A_{b \ldots}^{a \ldots}=\dot{A}_{b \ldots}^{a \ldots}+u^{n} \Gamma_{n k}^{a} A_{b \ldots}^{k \ldots}+\ldots-u^{n} \Gamma_{n b}^{k} A_{k \ldots}^{a \ldots}-\cdots,
$$

where $\dot{A}_{b \ldots}^{a \ldots}$ is the derivative of the components of $A$ along the $X$ direction,

$$
\dot{A}_{b \ldots}^{a \ldots}=u^{n} \frac{\partial A_{b \ldots}^{a \ldots}}{\partial x^{n}}+\dot{p}_{n} \frac{\partial A_{b \ldots}^{a \ldots}}{\partial p_{n}} .
$$

Note that the coordinate time derivative $\dot{p}_{a}$ and the covariant derivative $f_{a}$ are related by Eq. (2.6).

\footnotetext{
${ }^{3}$ In short, the partial derivative $\frac{\partial}{\partial p}$ is the derivative in a momentum direction with $x$ fixed and $\frac{\nabla}{\partial x}$ is the derivative in a configuration direction with $p$ parallel-transported. For more details, see Eqs. (A1) and (A2).
} 
For more details on the derivatives $\nabla_{X}, \frac{\nabla}{\partial x}, \frac{\partial}{\partial p}$ and the corresponding coordinate expressions we refer the reader to the Appendix.

\section{Derivative of Clifford fields}

The spacetime metric derivative can also be lifted to a derivative acting on phase-space fields with Dirac spinor indices. Namely, we are interested in Clifford objects, i.e., operators acting on Dirac spinors. These are generated by the abstract gamma matrices $\gamma^{a}$, obeying ${ }^{4}$

$$
\gamma^{a} \gamma^{b}+\gamma^{b} \gamma^{a}=2 g^{a b} \mathbb{1} .
$$

Thanks to this rule, any Clifford object $\phi$ can be represented by an inhomogeneous antisymmetric form $\omega=\sum_{r}^{r} \omega$,

$$
\phi=\sum_{r} \frac{1}{r !} \omega_{a_{1} \ldots a_{r}} \gamma^{a_{1} \ldots a_{r}} .
$$

Here, ${ }^{r} \omega$ are homogeneous rank- $r$ antisymmetric forms and

$$
\gamma^{a_{1} \ldots a_{r}}=\gamma^{\left[a_{1}\right.} \cdots \gamma^{\left.a_{r}\right]} .
$$

The covariant derivative on the Dirac bundle is induced from the spacetime metric derivative by the condition ${ }^{5}$

$$
\nabla_{n} \gamma^{a}=0
$$

Clearly, we can lift this derivative to act on the Cliffordvalued fields on the phase space in a similar way as we did for spacetime-tensor-fields. Namely, for $\Lambda(x, p)=$ $\lambda_{a_{1} \ldots a_{r}}(x, p) \gamma^{a_{1} \ldots a_{r}}(x)$ we simply get

$$
\nabla_{X} \Lambda=\left(\nabla_{X} \lambda_{a_{1} \ldots a_{r}}\right) \gamma^{a_{1} \ldots a_{r}} .
$$

To write down this covariant derivative in components, in addition to coordinates $x^{a}$, one has to introduce an orthonormal frame $e_{\hat{n}} \in \mathbf{T} M$ and the spinor frame $E_{\Upsilon}$ in such a way that the components ${ }^{6} \gamma^{\hat{a}}$ of the gamma matrices are constants. The covariant derivative of the Dirac spinor $\Phi$ expressed in the spinor frame then reads

$$
\nabla_{a} \Phi=\Phi_{, a}+\Sigma_{a} \Phi,
$$

with the connection coefficients $\Sigma_{a}$ uniquely determined in terms of the Ricci coefficients $\hat{\Gamma}_{a \hat{n}}^{\hat{m}}=\left(\nabla_{a} e_{\hat{n}}^{k}\right) e_{k}^{\hat{m}}$ by the standard relation

\footnotetext{
${ }^{4}$ In expressions with Clifford objects and Dirac spinors the Clifford multiplication is assumed. In components, it reduces to the standard matrix multiplication.

${ }^{5}$ To define the covariant derivative on Dirac spinors uniquely, the condition (2.16) must be supplemented by some further conditions reflecting the irreducibility and reality properties of $\gamma^{a}$. Thanks to Eq. (2.16) and rule (2.13), only the covariant derivative which annihilates the metric can be lifted to the Dirac bundle. In this context we always assume vanishing torsion, so the derivative on the tangent bundle must be the metric derivative.

${ }^{6}$ Components with respect to the frame $e_{\hat{n}}$ will be denoted with hatted indices. We will mostly skip the spinor indices (capital Greek letters), i.e., instead of $\gamma^{\hat{a} \Phi} \Psi$ we write just $\gamma^{\hat{a}}$. The matrix multiplication between Clifford objects and spinors is assumed.
}

$$
\Sigma_{a}=\frac{1}{4} \hat{\Gamma}_{a \hat{n}}^{\hat{m}} \gamma_{\hat{m}}^{\hat{n}}
$$

With these definitions the covariant derivative of the Clifford field $\Lambda(x, p)$ on the phase space is

$$
\nabla_{X} \Lambda=\dot{\Lambda}+\left[u^{n} \Sigma_{n}, \Lambda\right]
$$

where $\dot{\Lambda}$ is just an ordinary derivative along $X$ of components $\Lambda_{\Psi}^{\Phi}$.

\section{COVARIANT LAX EQUATION}

\section{A. Lax pair}

The Lax pair provides a useful tool for generating conserved quantities. The phase-space-valued matrices $L$ and $M$ form the Lax pair if they satisfy the Lax pair equation [1]

$$
\dot{\mathrm{L}}=[\mathrm{L}, \mathrm{M}] \text {. }
$$

Here, the dot is understood as the ordinary time derivative of each component of the matrix L. It follows that any scalar invariant formed from the matrix $L$ is a conserved quantity [cf. Eq. (1.3)].

It is customary to require that the Lax pair satisfies additional properties, especially that (i) Eq. (3.1) is equivalent to the equations of motion, and that (ii) the invariants of $L$ generate the maximal number of conserved quantities of the system. However, we will study Lax pairs without requiring these additional conditions. This is justified by realizing that the Lax pair equation (3.1) is linear in both $L$ and M. One can thus obtain a "more sophisticated" Lax pair as a direct sum of smaller matrices, each of which satisfy Eq. (3.1), and impose additional conditions only at the end, on the resulting pair.

\section{B. Lax tensor}

We shall now formulate an alternative covariant description of the Lax equation and clarify its relation to the standard Lax pair formulation. Using the above definition of the covariant phase-space derivative, we define the Lax tensor to be a covariantly conserved tensor field $L^{a}{ }_{b}(x, p)$, obeying the covariant Lax tensor equation

$$
\frac{\nabla}{d t} L_{b}^{a}=0
$$

Obviously, any scalar covariantly constructed ${ }^{7}$ from the Lax tensor or a set of Lax tensors is a constant of motion.

\footnotetext{
${ }^{7}$ The covariant derivative employed in the Lax equation (3.2) can be arbitrary. By covariant construction then we mean any operation which commutes with this derivative. Typical covariant operations are traces, contracted multiplication, or the determinant. If a tensor covariantly constant with respect to the chosen derivative is available, it can be used to construct the conserved scalar. Therefore, we typically choose the metric covariant derivative since then the metric "is available". However, one could use a different derivative; for example, if the Hamiltonian is $H=\frac{1}{2} p_{a} p_{b} k^{a b}$ with the "inverse mass" tensor $k^{a b}$ different from the metric $g^{a b}$.
} 
In particular, this is true for invariants constructed as traces [see Eq. (1.5)] of various powers of $L^{a}{ }_{b}$. Similarly to the Lax pair, the Lax tensor hence generates constants of motion.

Moreover, each Lax tensor defines a Lax pair. Indeed, in components, while using Eqs. (2.9) and (3.2) implies

$$
\dot{L}_{b}^{a}=L_{k}^{a} u^{n} \Gamma_{n b}^{k}-u^{n} \Gamma_{n k}^{a} L_{b}^{k} .
$$

The form of this equation is already very close to that of Eq. (3.1). However, the matrices forming the Lax pair must be defined as functions on the phase space. Therefore we have to eliminate the velocity $u$ using the first of the Hamilton equations (2.9). Thus the corresponding Lax pair matrices are

$$
\mathrm{L}=\left[L_{b}^{a}\right], \quad \mathrm{M}=\left[\frac{\partial H}{\partial p_{n}} \Gamma_{n b}^{a}\right] .
$$

Hence, Eq. (3.2) can be understood as a "covariant generalization" of the Lax pair equation (3.1); its coordinate form gives the Lax pair in the ordinary sense.

For motion in curved space governed by a given Hamiltonian, the covariant Lax tensor satisfying Eq. (3.2) and the ordinary Lax pair matrices (3.4) carry the same information. The Lax pair matrices are, however, coordinate-dependent. Under a change of coordinates the matrix $L$ transforms just by a trivial conjugation. However, the matrix $\mathrm{M}$ changes in a more complicated manner since the connection coefficients are involved.

\section{Clifford Lax tensor}

Any covariantly conserved ${ }^{8}$ antisymmetric form $\lambda_{a b \ldots}$ induces the Clifford field $\Lambda=\lambda_{a b \ldots} \gamma^{a b \ldots}$ satisfying

$$
\frac{\nabla}{d t} \Lambda=0 .
$$

We call $\Lambda$ a Clifford Lax tensor. In components, using Eqs. (2.18) and (3.5) implies

$$
\dot{\Lambda}=\left[\Lambda, u^{n} \Sigma_{n}\right] .
$$

In a way similar to the previous subsection, we find that the matrices

$$
\mathrm{L}=[\Lambda]=\left[\lambda_{\hat{a} \hat{b} \ldots} \gamma^{\hat{a} \hat{b} \ldots]}, \quad \mathrm{M}=\left[\frac{\partial H}{\partial p_{n}} \Sigma_{n}\right],\right.
$$

form a Lax pair satisfying Eq. (3.1).

In the next two sections we shall give a number of examples of (Clifford) Lax tensors for geodesic motion in curved spacetime. Whereas the following section concentrates on "trivial" examples in generic spacetimes (no enhanced symmetry is assumed), in Sec. V we discuss

\footnotetext{
${ }^{8}$ In the context of Clifford fields we always assume the metric covariant derivative since the abstract gamma matrices are then covariantly constant [see Eq. (2.16)], cf. footnote?
}

Lax tensors in special spacetimes admitting hidden symmetries. The motion of a charged particle in weakly charged Kerr-NUT spacetimes is discussed in Sec. VI.

\section{LAX TENSORS AND GEODESIC MOTION: TWO TRIVIAL EXAMPLES}

\section{A. Geodesic motion}

Geodesic motion with respect to the spacetime metric $g_{a b}$ is governed by the Hamiltonian

$$
H=\frac{1}{2 m} p_{a} g^{a b} p_{b} .
$$

The equations of motion (2.3) for canonical coordinates $x^{a}$, $p_{a}$ are

$$
\dot{x}^{a}=m^{-1} g^{a n} p_{n}, \quad \dot{p}_{a}=-\frac{1}{2 m} \frac{\partial g^{k l}}{\partial x^{a}} p_{k} p_{l} .
$$

These equations are equivalent to the geodesic equation $u^{n} \nabla_{n} u^{a}=0$ with the velocity $u^{a}=\dot{x}^{a}$ and $\nabla$ being the metric covariant derivative. They are also equivalent to the covariant equations on the phase space:

$$
p_{a}=m g_{a n} u^{n}, \quad \frac{\nabla}{d t} p_{a}=0 .
$$

\section{B. Lax tensor implying geodesic motion: Example I}

As a first example, let us study the simple tensor

$$
L^{a}{ }_{b}=g^{a n} p_{n} p_{b} .
$$

Thanks to Eq. (4.3), it is covariantly conserved and hence defines a Lax tensor.

In fact, we shall now prove that the existence of this Lax tensor is equivalent to the geodesic motion and hence it can be used as a starting point of the dynamics. In order to do that, we investigate the following problem: assuming that the covariant Lax equation (3.2) along an unknown Hamiltonian flow $X_{H}$ is satisfied for Eq. (4.4), does it imply that the Hamiltonian $H$ must generate geodesic motion?

Substituting Eq. (4.4) into Eq. (3.2), we obtain

$$
\frac{\nabla}{d t} L_{b}^{a}=f^{a} p_{b}+p^{a} f_{b}=0,
$$

which for generic momentum $p$ implies $f=0$. However, for the Hamiltonian flow, $f$ is given by Eq. (2.9), and we have

$$
f=-\frac{\nabla H}{\partial x}=0 .
$$

The Hamiltonian must thus be constructed only from momenta and covariantly constant spacetime tensors. In a generic curved spacetime the only covariantly constant spacetime tensors are constructed from the metric. Therefore, the Hamiltonian must be of the form 


$$
H=\frac{1}{2} h\left(p^{2}\right), \quad p^{2}=p_{a} p_{b} g^{a b},
$$

with $h$ being an arbitrary function. It implies the velocity $u^{a}=h^{\prime}\left(p^{2}\right) g^{a n} p_{n}$. Since $p^{2}$ is conserved, we have reproduced the geodesic equations of motion.

Thus the significance of the Lax tensor (4.4) lies in the fact that it implies geodesic motion. On the other hand, the only conserved quantities which can be obtained from this Lax tensor are functions of $p^{2}$ (which is, of course, the only conserved quantity for generic geodesic motion).

Using Eq. (3.4), the Lax tensor (4.4) defines the following Lax pair matrices:

$$
L_{b}^{a}=g^{a n} p_{n} p_{b}, \quad M_{b}^{a}=\frac{1}{m} p_{k} g^{k l} \Gamma_{l b}^{a} .
$$

We could ask the same question about equations of motion when starting with the ordinary Lax pair (4.8) satisfying Eq. (3.1). In this case the situation is slightly different. Using the explicit formula for the Christoffel coefficients $\Gamma_{l b}^{a}$ one can show that

$$
[L, M]_{b}^{a}=\left\{L_{b}^{a}, \frac{1}{2 m} p^{2}\right\},
$$

where the right-hand side is the Poisson bracket of the components of the Lax tensor $L$. Since $\dot{L}^{a}{ }_{b}=\left\{L^{a}{ }_{b}, H\right\}$, the Lax pair equation (3.1) implies the following condition on the Hamiltonian:

$$
\left\{g^{a n} p_{n} p_{b}, H-\frac{1}{2 m} p^{2}\right\}=0 .
$$

Obviously, the geodesic Hamiltonian $H=\frac{1}{2 m} p^{2}$ solves this condition. However, it is not clear if this is a unique solution.

\section{Lax tensor implying geodesic motion: Example II}

Allowing for Clifford-valued fields, it is possible to write an even simpler Lax tensor for the geodesic motion: ${ }^{9}$

$$
\Lambda=p_{a} \gamma^{a} .
$$

Similarly to Example I, this Lax tensor exists in a generic spacetime, it generates only conserved quantities which are functions of $p^{2}$, and the corresponding covariant Lax equation is equivalent to the geodesic equations of motion.

Using Eq. (3.7), the corresponding Lax pair matrices are

$$
\begin{aligned}
\mathrm{L} & =[\Lambda]=\left[p_{\hat{a}} \gamma^{\hat{a}}\right], \\
\mathrm{M} & =\left[\frac{1}{m} p^{n} \Sigma_{n}\right]=\left[\frac{1}{4 m} p^{n} \hat{\Gamma}_{n \hat{k} \hat{l}} \gamma^{\hat{k} \hat{l}}\right] .
\end{aligned}
$$

Similarly to Example I, it is not clear to us whether these Lax pair matrices imply geodesic motion. Namely, the

\footnotetext{
${ }^{9}$ This tensor is in some sense "Dirac's square root" of the Lax tensor (4.4); it can be obtained from the WKB approximation of the Dirac equation.
}

commutator in the Lax equation (3.1) can be simplified using properties of the gamma matrices

$$
\dot{p}_{\hat{a}} \gamma^{\hat{a}}=\frac{1}{m} p^{m} p_{\hat{n}} \hat{\Gamma}_{m \hat{a}}^{\hat{n}} \gamma^{\hat{a}} .
$$

We can thus eliminate the gamma matrices from both sides. Substituting the expression for the Ricci coefficients in terms of the derivatives of the components of $e_{\hat{n}}$, it is possible to show that

$$
\frac{1}{m} p^{m} p_{\hat{n}} \hat{\Gamma}_{m \hat{a}}^{\hat{n}}=\left\{p_{\hat{a}}, \frac{1}{2 m} p^{2}\right\} .
$$

Together with $\dot{p}_{\hat{a}}=\left\{p_{\hat{a}}, H\right\}$ it leads to an equation analogous to Eq. (4.10):

$$
\left\{p_{\hat{a}}, H-\frac{1}{2 m} p^{2}\right\}=0
$$

Beware however, that the frame component $p_{\hat{a}}$ of the momentum is not the canonical coordinate conjugated with $x^{a}$ [indeed, $p_{\hat{a}}=p_{n} e_{\hat{a}}^{n}(x)$ ], so Eq. (4.15) does not imply that $H-\frac{1}{2 m} p^{2}$ is independent of the coordinates. Similarly to the previous case, the geodesic Hamiltonian solves this condition, but it is an open question whether this solution is unique.

\section{LAX TENSORS AND HIDDEN SYMMETRIES}

Until now we have investigated rather trivial examples of Lax tensors, constructed from momenta and metricrelated quantities, and hence trivially conserved along the geodesic motion. Such objects are present in any generic spacetime. Now we shall turn to examples of Lax tensors present in spacetimes with enhanced symmetries, in particular admitting hidden symmetries of Killing and KillingYano tensors.

\section{A. Lax tensor from a conserved quantity}

Let us assume that, provided a given enhanced symmetry of the spacetime, an additional conserved quantity $E$ for geodesic motion is known. This, for example, incorporates the case of $E$ being generated from a Killing vector $\xi$, $E=\xi^{a} p_{a}$, or $E$ being generated from a Killing tensor $k$, $E=k^{a b \ldots} p_{a} p_{b} \ldots$ Of course, $L=E$ is a trivial onedimensional Lax tensor, which can be helpful, for example, if one constructs a larger Lax matrix as a sum of independent segments. One can also simply upgrade the Lax tensors (4.4) or (4.11) as

$$
L_{E}^{a}{ }_{b}=E p^{a} p_{b}, \quad \Lambda_{E}=E p_{a} \gamma^{a} .
$$

The corresponding Lax pair matrices follow from Eqs. (3.4) or (3.7). If more than one such constants are known, we may employ the method of spectral parameters [2] to combine the corresponding Lax tensors.

\section{B. Two kinds of Killing-Yano tensors}

The Killing-Yano (KY) tensor $\varphi_{a_{1} \ldots a_{r}}$ is [8] an antisymmetric form on the spacetime, the covariant derivative of 
which is determined by its antisymmetric part, i.e., by its exterior derivative $d \varphi$ :

$$
\nabla_{a} \varphi_{a_{1} \ldots a_{r}}=\nabla_{[a} \varphi_{\left.a_{1} \ldots a_{r}\right]} .
$$

On the other hand, the closed conformal Killing-Yano (CCKY) tensor $h_{a_{1} \ldots a_{r}}$ is an antisymmetric form on the spacetime, the covariant derivative of which is determined by its divergence $\xi_{a_{2} \ldots a_{r}}$ :

$$
\begin{aligned}
\nabla_{a} h_{a_{1} a_{2} \ldots a_{r}} & =r g_{a\left[a_{1}\right.} \xi_{\left.a_{2} \ldots a_{r}\right]}, \\
\xi_{a_{2} \ldots a_{r}} & =\frac{1}{D-r+1} \nabla_{n} h_{a_{2} \ldots a_{r}}^{n} .
\end{aligned}
$$

KY and CCKY tensors are related to each other through the Hodge duality: the Hodge dual of a KY form is a CCKY form and vice versa.

Both KY and CCKY tensors encode the so-called hidden symmetries: they exist only for special metrics and provide a rather rich structure to the geometry (see, e.g., Refs. [9-15] and references therein).

\section{Covariantly conserved tensors}

As one consequence of the above definitions, both KY and CCKY tensors define a tensorial quantity linear in momentum which is conserved along trajectories of the geodesic motion. Namely, for a KY tensor $\varphi$ and a CCKY tensor $h$ the quantities ${ }^{10}$

$$
\kappa=\varphi \cdot p, \quad \mu=h \wedge p,
$$

are conserved along geodesics,

$$
\frac{\nabla}{d t} \kappa=0, \quad \frac{\nabla}{d t} \mu=0 .
$$

The velocity is given by $u=\frac{1}{m} p$ and the momentum $p$ is conserved for the geodesic motion. Thus, thanks to Eq. (5.2), the derivative of $\kappa$ gives $\frac{1}{m} p^{k}\left(\nabla_{[k} \varphi_{\left.a_{1} \ldots a_{r-1} l\right]}\right) p^{l}=$ 0. Similarly, Eq. (5.3) implies $\frac{\nabla}{d t} h=\frac{1}{m} p \wedge h$ which vanishes when wedged with another $p$. Of course, both equations in Eq. (5.5) are equivalent through the Hodge duality: $\kappa=* \mu$ for $\varphi=* h$.

It is interesting to observe that the same information encoded in the forms $\kappa$ and $\mu$ is also encoded in the forms

$$
\Phi=\kappa \wedge p, \quad F=\mu \cdot p,
$$

respectively. Indeed, employing the identity

$$
(\alpha \cdot p) \wedge p+(\alpha \wedge p) \cdot p=p^{2} \alpha,
$$

which holds for any antisymmetric form $\alpha$, one can reconstruct $\kappa$ and $\mu$ from the quantities above:

\footnotetext{
${ }^{10}$ The dot "." denotes the contraction, $(\varphi \cdot p)_{a_{1} \ldots a_{r}}=$ $\varphi_{a_{1} \ldots a_{r} n} p^{n}$. Since we assume automatic rising of indices, it is essentially the scalar product.
}

$$
\kappa=\frac{1}{p^{2}} \Phi \cdot p, \quad \mu=\frac{1}{p^{2}} F \wedge p .
$$

Since the momentum is conserved for the geodesic motion, we thus obtain conserved quantities quadratic in momenta,

$$
\frac{\nabla}{d t} \Phi=0, \quad \frac{\nabla}{d t} F=0,
$$

which, however, carry the same information as those defined in Eq. (5.4). Note also the Hodge duality $\Phi=* F$ for $\varphi=* h$.

Finally, using Eq. (5.7) the form $F$ can be rewritten as

$$
F=(h \wedge p) \cdot p=p^{2} h-(h \cdot p) \wedge p,
$$

which in indices reads

$$
\begin{aligned}
F_{a_{1} a_{2} \ldots a_{r}}= & ((h \wedge p) \cdot p)_{a_{1} a_{2} \ldots a_{r}} \\
= & p^{2} h_{a_{1} a_{2} \ldots a_{r}}-h_{a_{1} a_{2} \ldots a_{r-1} n} p^{n} p_{a_{r}} \\
& -(-1)^{r-1} h_{a_{2} a_{3} \ldots a_{r} n} p^{n} p_{a_{1}}-\ldots \\
& -(-1)^{r-1} h_{a_{r} a_{1} \ldots a_{r-2} n} p^{n} p_{a_{r-1}} \\
= & p^{2} h_{n_{1} n_{2} \ldots n_{r}} P^{n_{1}}{ }_{a_{1}} P^{n_{2}} \ldots P_{2} \ldots P^{n_{r}},
\end{aligned}
$$

where we have introduced the projector

$$
P^{a}{ }_{b}=\delta^{a}{ }_{b}-p^{-2} p^{a} p_{b} .
$$

The form $F$ is thus (up to the prefactor $p^{2}$ ) the projection of the CCKY tensor $h$ onto a subspace orthogonal to the momentum $p$.

\section{Killing-Yano and Lax tensors}

All the conserved tensorial quantities $\kappa, \mu, \Phi$, and $F$ constructed from KY and CCKY tensors can be converted into Lax tensors using gamma matrices. For example, taking $\kappa$ and $\mu$, the corresponding Clifford objects read ${ }^{11}$

$$
\begin{aligned}
& \Lambda_{\varphi}=\gamma^{a_{1} \ldots a_{r-1}}(p \cdot \varphi)_{a_{1} \ldots a_{r-1}}, \\
& \Lambda_{h}=\gamma^{a_{1} \ldots a_{r+1}}(h \wedge p)_{a_{1} \ldots a_{r+1}} .
\end{aligned}
$$

They are covariantly conserved [Eq. (3.5)] and the corresponding Lax pair of matrices are given by Eq. (3.7). As mentioned above, the invariants of $\Lambda_{\varphi}$ span the same functional space as invariants of $\Phi$; in other words, the conserved scalar observables generated from the Lax tensor $\Lambda_{\varphi}$ are the same as those generated directly from $\Phi$, and similarly for $\Lambda_{h}$ and $F$.

Among all conserved quantities constructed from KY or CCKY tensors there are special cases which do not need to use Clifford objects and gamma matrices. Such a situation

\footnotetext{
${ }^{11}$ Similarly to Example II in the previous section, these Lax tensors can be understood as arising from the WKB approximation to the Dirac symmetry operators $K_{\varphi}$ and $M_{h}$ studied in Refs. [16,17].
} 
occurs if the conserved tensor under consideration is of rank-2. This includes:

(a) The CCKY tensor $h_{a}$ of rank 1, which is in fact a closed conformal Killing vector:

$$
\nabla_{a} h_{b}=\xi g_{a b}, \quad \xi=\frac{1}{D} \nabla_{n} h^{n} .
$$

The tensor $\mu$ is now of rank 2 , and hence directly generates the Lax tensor

$$
L^{a}{ }_{b}=h^{a} p_{b}-p^{a} h_{b} .
$$

The Lax pair matrices are given by

$$
\mathrm{L}=\left[L_{b}^{a}\right], \quad \mathrm{M}=\left[\frac{1}{m} p^{n} \Gamma_{n b}^{a}\right]
$$

cf. Eq. (3.4). The only independent constant of motion which can be obtained from this Lax tensor is $p^{2} h \cdot P \cdot h=p^{2} h^{2}-(p \cdot h)^{2}$.

(b) The CCKY tensor $h_{a b}$ of rank 2 generates the second-rank conserved quantity $F_{a b}$ given by Eqs. (5.6) or (5.11). The Lax tensor thus reads

$$
L^{a}{ }_{b}=F_{b}^{a}=p^{2} h_{b}^{a}-p^{a} p^{n} h_{n b}-h^{a n} p_{n} p_{b} .
$$

The Lax pair matrices are again given by Eq. (5.17). In the special case when $h_{a b}$ is nondegenerate, its very existence guarantees complete integrability of the geodesic motion; see the following subsection.

(c) The KY tensor $\varphi_{a b c}$ of rank 3 generates the Lax tensor

$$
L^{a}{ }_{b}=\varphi^{a}{ }_{b n} p^{n} .
$$

This case has been discussed in Refs. [3-7].

(d) The KY tensor $\varphi_{a b}$ of rank 2 generates the Lax tensor

$$
L_{b}^{a}=\Phi_{b}^{a}=\varphi^{a n} p_{n} p_{b}+p^{a} p^{n} \varphi_{n b} .
$$

The invariant generated from this Lax tensor is a function of the observable ${ }^{12} p_{a} p_{b} k^{a b}$, with $k^{a}{ }_{b}=\varphi^{a n} \varphi_{b n}$ being the Killing tensor of rank 2 associated with the KY tensor $\varphi_{a b}$.

(e) Any rank-2 tensor constructed from the momentum $p$ and quantities $\kappa$ and $\mu$ (for various $\mathrm{KY}$ forms $\varphi$ and CCKY forms $h$ ) by contractions and wedge operation. A simple interesting example is a "partial square" of the quantity $\kappa$, namely the Lax tensor

$$
L_{b}^{a}=\varphi^{a k l \ldots m} p_{m} \varphi_{b k l \ldots n} p^{n} .
$$

The trace of this Lax tensor gives the quadratic conserved observable $p_{a} p_{b} k^{a b}$, where the second-rank Killing tensor $k^{a b}$ is associated with the KY form $\varphi$ :

\footnotetext{
${ }^{12}$ Here we have used the fact that $p^{2}$ is also the conserved quantity, and we have canceled it out from $\operatorname{tr}\left(L^{2}\right)$.
}

$$
k^{a}{ }_{b}=\varphi^{a k l \ldots} \varphi_{b k l \ldots} .
$$

Another interesting possibility is to take a "square" of tensor (5.6),

$$
L^{a}{ }_{b}=F^{a k l \ldots} F_{b k l \ldots} .
$$

Depending on the rank of $\varphi$ this will generate a certain number of conserved quantities.

To summarize, the Lax tensors built from the KY and CCKY forms can be very fruitful. They can generate plenty of functionally independent invariants and they are thus very useful when investigating the conserved quantities.

\section{E. Kerr-NUT-anti-de Sitter spacetime}

A highly nontrivial example of the Lax tensor discusssed above can be found in the spacetime equipped with a nondegenerate CCKY tensor $h_{a b}$ of rank 2. It was proved in Refs. [18-20] that the existence of such a principal CCKY tensor determines the form of the metric up to a set of metric functions of a single argument. These functions can be fixed by the Einstein equation and the resulting vacuum (with cosmological constant) metric describes a generally rotating black hole in an arbitrary number of spacetime dimensions, also called the Kerr-NUT-anti-de Sitter spacetime [21-23].

In this case, the principal CCKY tensor $h_{a b}$ generates the covariantly conserved Lax tensor $F=(h \wedge p) \cdot p$ [cf. also Eq. (5.18)], which in $D=2 n+\varepsilon(\varepsilon=0,1)$ dimensions generates $n$ independent constants of geodesic motion [24-27]. It is possible to choose these constants in such a way that they are quadratic in momentum and hence are generated by rank-2 Killing tensors $k_{(j)}^{a b}$. Namely, it was shown in Ref. [25] that for any parameter $\beta$ the following identity holds:

$$
p^{2} \operatorname{det}\left(I+\sqrt{\beta} p^{-2} F\right)=\sum_{j=0}^{n} p_{a} p_{b} k_{(j)}^{a b} \beta^{j} .
$$

The left-hand side is a scalar expression, constructed just from the Lax tensor $F^{a}{ }_{b}$ and $p^{2}$, and hence is conserved for any $\beta$. The coefficients in the $\beta$ expansion are thus also conserved and can be read from the right-hand side of Eq. (5.24). They are quadratic in momentum, with the $k_{(j)}$ being Killing tensors. ${ }^{13}$

Moreover, the discussed spacetime also admits $n+\varepsilon$ explicit (Killing vector) symmetries which supply additional $n+\varepsilon$ conserved quantities. All these $D$ conserved quantities are in involution and the system is completely integrable. The existence of the quadratic conserved quantities encoded in the Lax tensor is also a

\footnotetext{
${ }^{13}$ For $j=0$ the Killing tensor reduces to the metric, $k_{(0)}^{a b}=g^{a b}$. The $n$th Killing tensor vanishes in even dimensions, whereas it is reducible to a square of one of the Killing vectors in odd dimensions. For $j=0, \ldots, n-1$ the Killing tensors are irreducible, thus giving $n$ quadratic constants of motion.
} 
starting point for showing that the Hamilton-Jacobi, KleinGordon, and Dirac equations separate in these spacetimes [16,17,28-30]. (See also Refs. [31,32] for separability of certain gravitational perturbations.)

\section{MOTION OF A CHARGED PARTICLE}

It was shown in Ref. [33] that the motion of a charged particle in the special test electromagnetic field in the background of Kerr-NUT spacetimes in all dimensions is also completely integrable. Here we demonstrate that the conserved quantities quadratic in momentum can be found using the Lax tensor method.

The electromagnetic field under investigation is given by the vector potential $A$ proportional to the primary Killing vector $\xi$ of the Kerr-NUT geometry. ${ }^{14}$ The primary Killing vector $\xi$ is a divergence of the principal CCKY tensor $h_{a b}$, $\xi^{a}=\frac{1}{D-1} \nabla_{c} h^{c a}$ [cf. Eq. (5.3)]. We will write $q A_{a}=e \xi_{a}$, where $q$ is the charge of the particle and $e$ is a constant combining both the charge and the strength of the field. The motion of the charged particle is thus governed by the Hamiltonian:

$$
H=\frac{1}{2 m}\left(p_{a}-e \xi_{a}\right) g^{a b}\left(p_{b}-e \xi_{b}\right) .
$$

The relation between the velocity and momentum can be read from the Hamiltonian flow (2.9):

$$
u^{a}=\frac{1}{m}\left(p^{a}-e \xi^{a}\right) .
$$

The covariant derivative of the momenta is

$$
\frac{\nabla}{d t} p_{a}=\frac{e}{m}\left(\nabla_{a} \xi_{n}\right)\left(p^{n}-e \xi^{n}\right) .
$$

Substituting Eq. (6.2) and using the Killing vector condition $\nabla_{a} \xi_{n}=-\nabla_{n} \xi_{a}$, one gets $\frac{\nabla}{d t} p_{a}=-e u^{n} \nabla_{n} \xi_{a}=$ $-e \frac{\nabla}{d t} \xi_{a}$, i.e.,

$$
\frac{\nabla}{d t}(p+e \xi)=0 .
$$

Now we can prove that the quantity

$$
\mu=h \wedge(p+e \xi)
$$

is covariantly conserved. Indeed, using Eqs. (6.4), (5.3), and (6.2), we get

$$
\frac{\nabla}{d t} \mu=(u \wedge \xi) \wedge(p+e \xi)=0 .
$$

It follows that

$$
F=\mu \cdot(p+e \xi)
$$

is also covariantly conserved. Being the tensor of rank 2 , this is the covariant Lax tensor for our system. It is

\footnotetext{
${ }^{14}$ The condition of vanishing electric current for such an electromagnetic field requires the cosmological constant to be set equal to zero, cf. Ref. [33].
}

constructed in a similar way as the Lax tensor from the previous subsection, only with the substitution $p \rightarrow p+$ $e \xi$. The scalars generated from $F$ can thus be read again from Eq. (5.24). The quadratic constants of motion are

$$
\tilde{K}_{(j)}=\left(p_{a}+e \xi_{a}\right)\left(p_{b}+e \xi_{b}\right) k_{j}^{a b} .
$$

These differ from the constants $K_{(j)}$ introduced in Ref. [33], but only by terms $L_{(j)}$ linear in momentum, which are also conserved:

$$
\begin{gathered}
K_{(j)}=\left(p_{a}-e \xi_{a}\right)\left(p_{b}-e \xi_{b}\right) k_{j}^{a b}=\tilde{K}_{(j)}-4 e L_{(j)}, \\
L_{(j)}=p_{a} l_{(j)}^{a}=p_{a} k_{(j)}^{a n} \xi_{n} .
\end{gathered}
$$

The conservation of $L_{(j)}$ follows from the fact that $l_{(j)}^{a}=$ $k_{(j)}^{a n} \xi_{n}$ are Killing vectors [25]. It was demonstrated in Ref. [33] that the conserved quantities $K_{(j)}$ and $L_{(j)}$ are all in involution.

\section{SUMMARY}

The Lax pair formalism provides an elegant and effective description of special dynamical systems with enhanced symmetries. In particular, the existence of the Lax pair, defined by Eq. (1.2), enables one to generate constants of motion by simple algebraic operations, e.g., Eq. (1.3).

In this paper we have provided an alternative, covariant formulation of the Lax formalism. This is based on the covariant (Clifford) Lax tensor, where the Lax equation is formulated as a covariant conservation of this tensor, Eqs. (3.2) and (3.5). In both instances the existence of the Lax tensor enables one to generate constants of motion, which are determined as invariants constructed from the object, e.g., Eq. (1.5). We have further demonstrated that the ordinary Lax pair matrices follow from the covariant Lax formalism, the relation being given by Eqs. (3.4) and (3.7).

To illustrate the derived formulas, we have concentrated on the problem of particle motion in curved spacetime. In this case we were able to provide a number of examples of (Clifford) Lax tensors. In particular, we have concentrated on manifolds with enhanced symmetry, admitting hidden symmetries of Killing-Yano tensors, in which case the examples of Lax tensors are highly nontrivial. One of the Lax tensors discussed was proven to be responsible for the complete integrability of geodesic motion in rotating black hole spacetimes in all dimensions just a few years ago. We have demonstrated for the first time that the conserved quantities for motion of a charged particle in the aligned test electromagnetic field on the same (vacuum) black hole background can also be generated using the Lax tensor (6.7).

It remains an interesting open question whether any of the Lax tensors discussed here will find further physical applications in the future. Another interesting question is whether some examples of the geometric Lax tensors can be found for other integrable (supersymmetric) systems, e.g., Ref. [34]. 


\section{ACKNOWLEDGMENTS}

V.F. thanks the Natural Sciences and Engineering Research Council of Canada and the Killam Trust for financial support. P. K. was supported by Grants GAČR 202/09/0772 and GAČR P203/12/0118. D. K. and P. K. acknowledge hospitality at the University of Alberta where this work was partially done. M. C. is partially funded by Fapemig under the project CEX APQ 2324-11.

\section{APPENDIX: DERIVATIVES ON THE PHASE SPACE WITH COTANGENT BUNDLE STRUCTURE}

In this Appendix we discuss the structure of the cotangent bundle phase space ${ }^{15} P=\mathbf{T}^{*} M$ in more detail. We show that the covariant derivative on the configuration space $M$ induces a covariant splitting of the phase-space quantities into quantities related to the configuration space. It naturally replaces standard coordinate expressions in a coordinate-independent way.

\section{Derivatives along position and momentum directions}

First, we introduce covariant partial derivatives of a scalar observable along the position and momentum directions. The derivative in the momentum direction $f_{a}$ (i.e., changes along a curve $p_{a} \rightarrow p_{a}+\varepsilon f_{a}, x$ fixed) is simple, since the space of momenta at fixed $x$ is linear. We define

$$
f_{a} \frac{\partial F}{\partial p_{a}}=\left.\frac{d}{d \varepsilon} F(x, p+\varepsilon f)\right|_{\varepsilon=0} .
$$

Thanks to ultralocality in $f_{a}$ we can tear off $f_{a}$ to obtain the derivative operator $\frac{\partial}{\partial p_{a}}$ (with one contravariant configurationspace index) acting on the scalar phase-space observables. Such a derivative operator also defines a mixed tensor $\frac{\partial^{A}}{\partial p_{a}} \in$ $\mathbf{T} P \otimes \mathbf{T} M$. It is actually the tensor identifying the tangent space of the cotangent fibre $\mathbf{T}\left(\mathbf{T}_{x}^{*} M\right)$ with the cotangent fibre $\mathbf{T}_{x}^{*} M$ itself.

The derivative along a position direction with momentum fixed is more involved. Moving from one position to another one changes the cotangent fibre and it has to be clarified what "fixed momentum" means. A natural solution is given in terms of a spacetime-covariant derivative ${ }^{16}$ $\nabla$. The covariant derivative defines "fixed momentum" to be the parallel-transported momentum. Let $x_{\varepsilon}$ be a spacetime curve in the $u^{a}$ direction and $\bar{p}_{\varepsilon}$ be the paralleltransported momentum along this curve. Then we can write

$$
u^{a} \frac{\nabla_{a} F}{\partial x}=\left.\frac{d}{d \varepsilon} F\left(x_{\varepsilon}, \bar{p}_{\varepsilon}\right)\right|_{\varepsilon=0} .
$$

\footnotetext{
${ }^{15}$ We use capital Latin indices for the phase-space tensors, and in this Appendix we write these indices explicitly. The material presented here partially follows and partially generalizes the Appendix of Ref. [26].

${ }^{16}$ It can be an arbitrary covariant derivative. Of course, in most cases it is useful to chose the metric derivative.
}

Again, it defines the phase-space derivative $\frac{\nabla_{a}}{\partial x}$ with one covariant configuration-space index and the mixed tensor $\frac{\nabla_{a}^{A}}{\partial x} \in \mathbf{T} P \otimes \mathbf{T}^{*} M$. The last quantity is the tensor which makes a horizontal lift of the configuration-space vector $u^{a}$ to the horizontal phase-space vector $u^{a} \frac{\nabla_{a}^{A}}{\partial x}$ (see Fig. 1).

The action of these derivatives on an observable of the form $A(x, p)=\alpha^{a b \ldots(x)} p_{a} p_{b} \ldots$ is

$$
\begin{gathered}
\frac{\nabla_{n} A}{\partial x}=\nabla_{n} \alpha^{a b \cdots}(x) p_{a} p_{b} \cdots \\
\frac{\partial A}{\partial p_{n}}=\alpha^{n b \cdots}(x) p_{b} \cdots+\alpha^{a n \cdots}(x) p_{a} \cdots+\cdots
\end{gathered}
$$

$$
P=\mathbf{T}^{*} M
$$

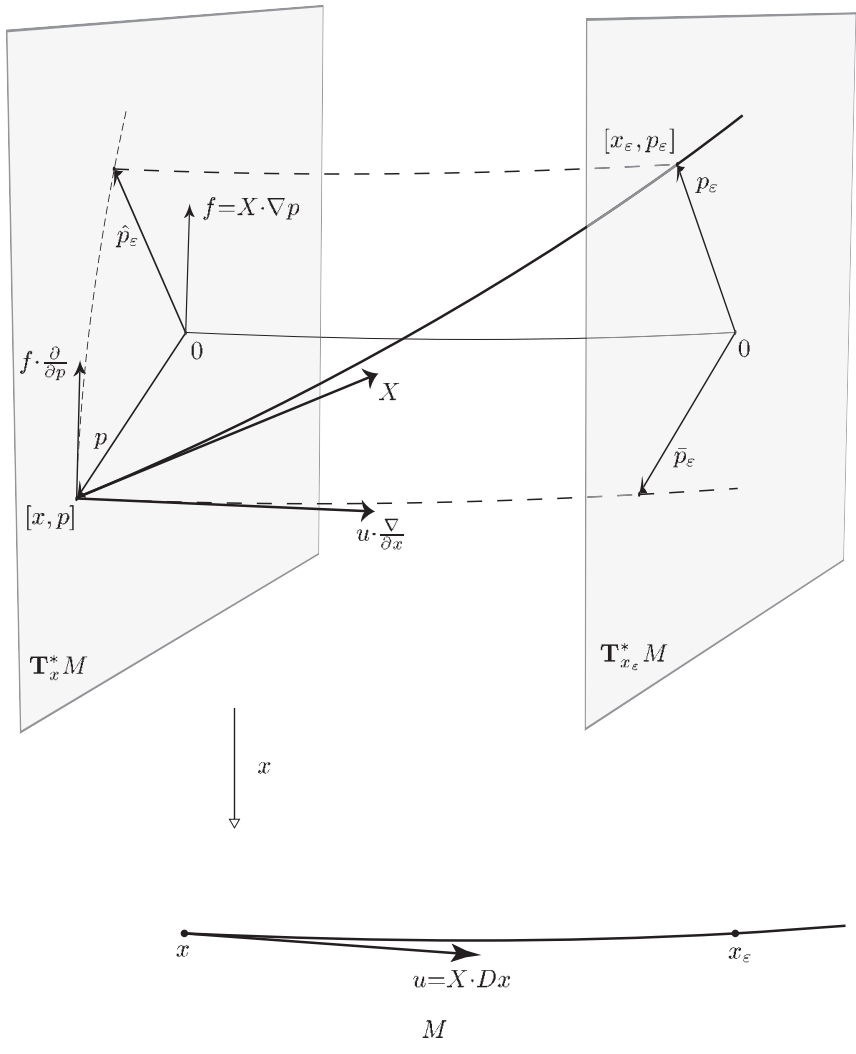

FIG. 1. Splitting of the phase-space direction $X$. In this figure we illustrate the splitting of the phase-space direction $X$ into its position and momentum parts. Let $\left[x_{\varepsilon}, p_{\varepsilon}\right]$ be a curve starting at $[x, p]$ to which $X$ is tangent. Its corresponding position and momentum parts are $x_{\varepsilon}$ and $p_{\varepsilon}$. The dashed curve $\bar{p}_{\varepsilon}$ is the parallel transport of the initial momentum $p$ along $x_{\varepsilon}$. The shortdashed curve $\hat{p}_{\varepsilon}$ laying in the cotangent fibre $\mathbf{T}_{x}^{*} M$ is obtained as the parallel transport of $p_{\varepsilon}$ along $x_{\varepsilon}$ back to the point $x$. Various vectors defined in the text are tangent vectors to these curves. The phase-space vector $X$ is tangent to $\left[x_{\varepsilon}, p_{\varepsilon}\right]$, its position direction $u$ is tangent to $x_{\varepsilon}$, and the momentum part $f$ is the derivative of $\hat{p}_{\varepsilon}$, which is understood to be a cotangent vector. In other words, $f$ is the covariant derivative of $p_{\varepsilon}$ along $x_{\varepsilon}$, i.e., along the direction $u$. The formula (A14) splits $X$ into $u \cdot \frac{\nabla}{\partial x}$, which is tangent to $\bar{p}_{\varepsilon}$, and into $f \cdot \frac{\partial}{\partial p}$, which is tangent to $\hat{p}_{\varepsilon}$. 
The action on a general observable can be written explicitly using linearity, the Leibnitz product rule, and the chain rule.

The mixed tensors $\frac{\nabla_{a}^{A}}{\partial x}$ and $\frac{\partial^{A}}{\partial p_{a}}$ are a covariant generalization of the phase-space coordinate vectors ${ }^{17} \frac{\partial^{A}}{\partial x^{a}}$ and $\frac{\partial^{A}}{\partial p_{a}}$ associated with the canonical coordinates $\left(x^{a}, p_{a}\right)$. Therefore, one can expect that natural symplectic quantities can be written using these covariant tensors. Namely, the inverse symplectic structure $\Omega^{-1 A B}$ (such that $\Omega_{A N} \Omega^{-1 B N}=\delta_{A}^{B}$ ), the Poisson brackets, and the Hamiltonian flow $X_{H}^{A}=\Omega^{-1 A N} d_{N} H$ are

$$
\begin{array}{r}
\Omega^{-1 A B}=\frac{\nabla_{n}^{A}}{\partial x} \frac{\partial^{B}}{\partial p_{n}}-\frac{\partial^{A}}{\partial p_{n}} \frac{\nabla_{n}^{B}}{\partial x}+p_{n} T_{k l}^{n} \frac{\partial^{A}}{\partial p_{k}} \frac{\partial^{B}}{\partial p_{l}}, \\
\{F, G\}=\frac{\nabla_{n} F}{\partial x} \frac{\partial G}{\partial p_{n}}-\frac{\partial F}{\partial p_{n}} \frac{\nabla_{n} G}{\partial x}+p_{n} T_{k l}^{n} \frac{\partial F}{\partial p_{k}} \frac{\partial G}{\partial p_{l}}, \\
X_{H}^{A}=\frac{\partial H}{\partial p_{n}} \frac{\nabla_{n}^{A}}{\partial x}-\frac{\nabla_{n} H}{\partial x} \frac{\partial^{A}}{\partial p_{n}}-p_{n} \frac{\partial H}{\partial p_{k}} T_{k l}^{n} \frac{\partial^{A}}{\partial p_{l}} .
\end{array}
$$

Here, $T_{k l}^{n}$ is the torsion of $\nabla$. For vanishing torsion $(T=0)$ the expressions resemble the standard coordinate formulas.

\section{Dual quantities and splitting of the phase-space direction}

We can also construct dual quantities $D_{A}^{n} x$ and $\nabla_{A} p_{n}$ which correspond to coordinate forms $d_{A} x^{a}$ and $d_{A} p_{a}$. We require the duality conditions

$$
\begin{array}{ll}
\frac{\nabla_{a}^{N}}{\partial x} D_{N}^{b} x=\delta_{a}^{b}, & \frac{\partial^{N}}{\partial p_{a}} \nabla_{N} p_{b}=\delta_{b}^{a}, \\
\frac{\nabla_{a}^{N}}{\partial x} \nabla_{N} p_{b}=0, & \frac{\partial^{N}}{\partial p_{a}} D_{N}^{b} x=0 .
\end{array}
$$

The completeness relation reads

$$
D_{A}^{n} x \frac{\nabla_{n}^{B}}{\partial x}+\nabla_{A} p_{n} \frac{\partial^{B}}{\partial p_{n}}=\delta_{A}^{B} .
$$

The symplectic structure $\Omega_{A B}$ and the symplectic potential $\theta_{A}=-p_{n} d_{A} x^{n}$ have a familiar form (except for the anomalous torsion term):

$\Omega_{A B}=D_{A}^{n} x \nabla_{B} p_{n}-\nabla_{A} p_{n} D_{B}^{n} x-p_{n} T_{k l}^{n} D_{A}^{k} x D_{B}^{l} x$,

\footnotetext{
${ }^{17}$ The notation here is a partially mistreats the difference between the covariant nature of the mixed tensors and the coordinatedependent nature of the coordinate tensors. For example, both indices in $\frac{\nabla_{a}^{A}}{\partial x}$ are tensor indices and could be understood as abstract indices, independent of the chosen coordinates. Similarly for the phase-space index $A$ in the coordinate vector $\frac{\partial^{A}}{\partial x^{n}}$. However, the index $n$ here is not a tensor index; it just labels which coordinate tensor we are choosing. To make this distinction clear, one should distinguish the abstract and coordinate indices as, e.g., in Ref. [35]. We decided not to do so and to let the reader distinguish tensorial and coordinate indices based on the context. In general, in expressions not involving explicitly chosen coordinates all indices are tensorial. If the coordinates are involved, the combinations $x^{a}$ and $p_{a}$ usually indicate the coordinate indices.
}

$$
\theta_{A}=-p_{n} D_{A}^{n} x
$$

These dual mixed tensors also define the splitting of a phase-space vector $X^{A}$ into the configuration-space quantities $u^{a}$ and $f_{a}$ discussed in Sec. II near Eq. (2.5). Such splitting is based on the observation that the covariant derivative splits the tangent fibre $\mathbf{T}_{[x, p]} P$ into horizontal and vertical subspaces. ${ }^{18}$ The configuration direction $u^{a}$ is the projection of $X^{A}$ onto the spacetime (thus encoding the horizontal part) and the momentum direction $f_{a}$ is the vertical part of $X^{A}$. These two parts can be written using $D x$ and $\nabla p$ (see Fig. 1).

Indeed, the tensor $D x$ is just the differential of the projection map $x:[x, p] \rightarrow x$ from the phase space to the configuration space. Given a phase-space vector $X^{A}$, its shadow on the configuration space is thus

$$
u^{a}=X^{A} D_{A}^{a} x \text {. }
$$

On other hand, the quantity $\nabla p$ is the projector of a phasespace vector $X^{A}$ on its vertical part $f_{a}$ :

$$
f_{a}=X^{A} \nabla_{A} p_{a} .
$$

The completeness relation (A9) together with Eqs. (A12) and (A13) gives

$$
X^{A}=u^{n} \frac{\nabla_{n}^{A}}{\partial x}+f_{n} \frac{\partial^{A}}{\partial p_{n}},
$$

(cf. Fig. 1) and the derivative of the scalar observable $F(x, p)$ along the phase-space direction $X^{A}$ is thus

$$
X^{A} d_{A} F=u^{n} \frac{\nabla_{n} F}{\partial x}+f_{n} \frac{\partial F}{\partial p_{n}} .
$$

\section{The covariant derivative induced on the phase space}

The covariant derivative of the phase-space fields with configuration-space indices introduced in Sec. II can be understood as a generalization of the formula (A15) to tensor fields. The derivative $\frac{\partial}{\partial p_{n}}$ acting in the momentum directions has the same definition [Eq. (A1)]. The definition of the derivative $\frac{\nabla_{a}}{\partial x}$ in the configuration direction changes by employing just the covariant derivative ${ }^{19}$ in the definition (A2):

$$
u^{n} \frac{\nabla_{n} A_{b \ldots}^{a \ldots}}{\partial x}=\left.\frac{\nabla}{d \varepsilon} A_{b \ldots}^{a \ldots .}\left(x_{\varepsilon}, \bar{p}_{\varepsilon}\right)\right|_{\varepsilon=0} .
$$

The action of the derivatives $\frac{\nabla_{a}}{\partial x}$ and $\frac{\partial}{\partial p_{n}}$ on the tensor field $A_{b \ldots}^{a \ldots}(x, p)=\alpha_{b \ldots \ldots}^{a \ldots k l \ldots} p_{k} p_{l} \ldots$ is analogous to rules (A3) and (A4), just with additional indices involved.

\footnotetext{
${ }^{18}$ The horizontal subspace of $\mathbf{T}_{[x, p]} P$ gives the directions of parallel-transported momenta, and the vertical subspace is tangent to the fibre $\mathbf{T}_{x}^{*} M$.

${ }^{19}$ Since $A_{b . \ldots}^{a \ldots}\left(x_{\varepsilon}, p_{\varepsilon}\right)$ is just $\varepsilon$-dependent, it can be understood as a tensor field along the configuration curve $x_{\varepsilon}$, and just the standard covariant derivative in the configuration space is involved on the right-hand side of the definition (A16).
} 
The covariant derivative along a general phase-space direction $X^{A}$ [split as in Eq. (A14)] acting on a field $A_{b \ldots}^{a \ldots}(x, p)$ is then given [by the generalization of Eq. (A15)] as

$$
\frac{\nabla}{d \varepsilon} A_{b \ldots}^{a \ldots} \equiv \nabla_{X} A_{b \ldots}^{a \ldots}=u^{n} \frac{\nabla_{n}}{\partial x} A_{b \ldots}^{a \ldots}+f_{n} \frac{\partial}{\partial p_{n}} A_{b \ldots}^{a \ldots} .
$$

This was already mentioned in Eq. (2.7). In particular, the derivatives of the pure configurations field $A_{b . . .}^{a \ldots}(x)$ and of the momentum field $p_{a}$ are given by rules (i) and (ii) in Sec. II.

Since the dependence on $X^{A}$ is ultralocal, it is possible to also define the covariant differential $\nabla_{N} A_{b \ldots}^{a \ldots}$, namely

$$
\nabla_{N} A_{b \ldots}^{a \ldots}=D_{N}^{n} x \frac{\nabla_{n}}{\partial x} A_{b \ldots}^{a \ldots}+\nabla_{N} p_{n} \frac{\partial}{\partial p_{n}} A_{b \ldots}^{a \ldots} .
$$

\section{Relation to canonical coordinates}

In the definitions above we have not used any specific choice of the coordinates. However, the introduced formalism can be easily accommodated to such a choice. If we choose configuration-space coordinates $x^{a}$, one can define the "coordinate derivative" $\partial$ by the conditions

$$
\partial d x^{b}=0, \quad \partial \frac{\partial}{\partial x^{b}}=0 .
$$

This is a torsion-free covariant derivative (of course, depending on the choice of coordinates). The difference tensor between $\nabla$ and $\partial$ is given by the connection coefficients $\Gamma_{a b}^{n}$.

We can use the coordinate derivative $\partial$ instead of $\nabla$ in all expressions above. It leads to standard coordinate expressions: the Eq. (A10) reduces to Eq. (2.1), the expression (A6) to Eq. (2.2), etc. In the coordinate case we also use the more common notation $\frac{\partial}{\partial x^{a}}$ instead of $\frac{\partial a}{\partial x}$. The induced coordinate derivative on the phase space along a phasespace direction $X^{A}$ is denoted just by a dot:

$$
\dot{A}_{b \ldots}^{a \ldots}=\frac{\partial}{\partial \varepsilon} A_{b \ldots}^{a \ldots}=\partial_{X} A_{b \ldots}^{a \ldots .} .
$$

The splitting of the phase-space direction $X^{A}$ depends on the choice of the covariant derivative. For the coordinate derivative this splitting gives

$$
u^{a}=\xi^{A} D_{A}^{a} x, \quad \dot{p}_{a}=\xi^{A} \partial_{A} p_{a} .
$$

Clearly, $\dot{p}_{a}$ are just derivatives of the components of $p$ along $X^{A}$, which justifies the dot notation.

Let $\left[x_{\varepsilon}, p_{\varepsilon}\right]$ be a phase-space curve with tangent vector $X$. Then, $f_{a}=X^{A} \nabla_{A} p_{a}$ can be understood as the standard configuration-space covariant derivative of $p_{\varepsilon}$ along the spacetime curve $x_{\varepsilon}$ (cf. Fig. 1). Similarly, $\dot{p}_{a}=X^{A} \partial_{A} p_{a}$ is the coordinate derivative of $p_{\varepsilon}$ along $x_{\varepsilon}$. We can thus use the ordinary relation between covariant and coordinate derivatives to obtain

$$
f_{a}=\dot{p}_{a}-u^{k} \Gamma_{k a}^{l} p_{l}
$$

[cf. Eq. (2.6)]. Here, the velocity $u^{k}$ is tangent to the curve $x_{\varepsilon}$.

Tearing off the phase-space vector $X^{A}$, we obtain

$$
\nabla_{A} p_{n}=\partial_{A} p_{n}-D_{A}^{k} x \Gamma_{k n}^{l} p_{l} .
$$

The duality relations (A8) imply

$$
\frac{\nabla_{n}^{A}}{\partial x}=\frac{\partial^{A}}{\partial x^{n}}+p_{k} \Gamma_{n l}^{k} \frac{\partial^{A}}{\partial p_{l}} .
$$

The action on a scalar observable reads

$$
\frac{\nabla_{n} F}{\partial x}=\frac{\partial F}{\partial x^{n}}+p_{k} \Gamma_{n l}^{k} \frac{\partial F}{\partial p_{l}} .
$$

The generalization to the action on tensor fields adds only standard terms for each tensor index:

$$
\begin{aligned}
\frac{\nabla_{n}}{\partial x} A_{b \ldots}^{a \ldots}= & \frac{\partial A_{b \ldots}^{a \ldots}}{\partial x^{n}}+p_{k} \Gamma_{n l}^{k} \frac{\partial A_{b \ldots}^{a \ldots}}{\partial p_{l}}+\Gamma_{n k}^{a} A_{b \ldots}^{k \ldots} \\
& +\cdots-\Gamma_{n b}^{k} A_{k \ldots}^{a \ldots}-\cdots .
\end{aligned}
$$

If we substitute Eqs. (A22) and (A26) into Eq. (A17), we obtain the coordinate expression for the covariant derivative on the phase space:

$$
\frac{\nabla}{\partial \varepsilon} A_{b \ldots}^{a \ldots}=\dot{A}_{b \ldots}^{a \ldots}+u^{n} \Gamma_{n k}^{a} A_{b \ldots}^{k \ldots}+\cdots-u^{n} \Gamma_{n b}^{k} A_{k \ldots}^{a \ldots}-\cdots
$$

Here, the coordinate derivative $\dot{A}_{b \ldots}^{a \ldots}$ splits as

$$
\dot{A}_{b \ldots}^{a \ldots}=u^{n} \frac{\partial A_{b \ldots}^{a \ldots}}{\partial x^{n}}+\dot{p}_{n} \frac{\partial A_{b \ldots}^{a \ldots}}{\partial p_{n}} .
$$

[1] P.D. Lax, Commun. Pure Appl. Math. 21, 467 (1968).

[2] O. Babelon, D. Bernard, and M. Talon, Introduction to Classical Integrable Systems (Cambridge University Press, Cambridge, England, 2003).

[3] K. Rosquist, arXiv:gr-qc/9410011.
[4] K. Rosquist and M. Goliath, Gen. Relativ. Gravit. 30, 1521 (1998).

[5] M. Karlovini and K. Rosquist, Gen. Relativ. Gravit. 31, 1271 (1999).

[6] D. Baleanu and A. K. Karasu, Mod. Phys. Lett. A 14, 2587 (1999). 
[7] D. Baleanu and S. Baskal, Mod. Phys. Lett. A 15, 1503 (2000).

[8] K. Yano, Ann. Math. 55, 328 (1952).

[9] U. Semmelmann, arXiv:math/0206117.

[10] G. Papadopoulos, Classical Quantum Gravity 25, 105016 (2008).

[11] V.P. Frolov and D. Kubizňák, Classical Quantum Gravity 25, 154005 (2008).

[12] Y. Yasui and T. Houri, Prog. Theor. Phys. Suppl. 189, 126 (2011).

[13] O. Santillan, J. Math. Phys. (N.Y.) 53, 043509 (2012).

[14] M. Visinescu and G. E. Vilcu, SIGMA 8, 058 (2012).

[15] M. Cariglia, arXiv:1209.6406.

[16] M. Cariglia, P. Krtous, and D. Kubiznak, Phys. Rev. D 84, 024004 (2011).

[17] M. Cariglia, P. Krtous, and D. Kubiznak, Phys. Rev. D 84, 024008 (2011).

[18] T. Houri, T. Oota, and Y. Yasui, Phys. Lett. B 656, 214 (2007).

[19] P. Krtouš, V. P. Frolov, and D. Kubizňák, Phys. Rev. D 78, 064022 (2008).

[20] T. Houri, T. Oota, and Y. Yasui, Phys. Lett. B 666, 391 (2008).

[21] R. C. Myers and M. J. Perry, Ann. Phys. (N.Y.) 172, 304 (1986).
[22] G. W. Gibbons, H. Lü, D. N. Page, and C. N. Pope, Phys. Rev. Lett. 93, 171102 (2004).

[23] W. Chen, H. Lü, and C. N. Pope, Classical Quantum Gravity 23, 5323 (2006).

[24] D. N. Page, D. Kubižnák, M. Vasudevan, and P. Krtouš, Phys. Rev. Lett. 98, 061102 (2007).

[25] P. Krtouš, D. Kubizňák, D. N. Page, and V. P. Frolov, J. High Energy Phys. 02 (2007) 004.

[26] P. Krtouš, D. Kubizňák, D. N. Page, and M. Vasudevan, Phys. Rev. D 76, 084034 (2007).

[27] T. Houri, T. Oota, and Y. Yasui, J. Phys. A 41, 025204 (2008).

[28] V.P. Frolov, P. Krtouš, and D. Kubizňák, J. High Energy Phys. 02 (2007) 005.

[29] A. Sergyeyev and P. Krtouš, Phys. Rev. D 77, 044033 (2008).

[30] T. Oota and Y. Yasui, Phys. Lett. B 659, 688 (2008).

[31] H. K. Kunduri, J. Lucietti, and H. S. Reall, Phys. Rev. D 74, 084021 (2006).

[32] T. Oota and Y. Yasui, Int. J. Mod. Phys. A 25, 3055 (2010).

[33] V.P. Frolov and P. Krtous, Phys. Rev. D 83, 024016 (2011).

[34] A. Galajinsky, Phys. Rev. D 85, 085002 (2012).

[35] R. Penrose and W. Rindler, Spinors and Space-Time (Cambridge University Press, Cambridge, England, 1986). 